\title{
Philosophiques
}

\section{L’articulation du général et du particulier : une approche méthodologique dans le champ des sciences sociales}

\section{Roberto Miguelez}

Volume 11, numéro 2, octobre 1984

Égalité, justice et différence

URI : https://id.erudit.org/iderudit/203257ar

DOI : https://doi.org/10.7202/203257ar

Aller au sommaire du numéro

Éditeur(s)

Société de philosophie du Québec

ISSN

0316-2923 (imprimé)

1492-1391 (numérique)

Découvrir la revue

Citer cet article

Miguelez, R. (1984). L'articulation du général et du particulier : une approche méthodologique dans le champ des sciences sociales. Philosophiques, 11(2),

251-276. https://doi.org/10.7202/203257ar
Résumé de l'article

La question de l'articulation du général et du particulier n'a pas qu'une dimension épistémologique, elle commande aussi la résolution du problème du rapport entre théorie et pratique éclairée, et ceci dans la mesure où la pratique s'exerce toujours sur des processus ou des phénomènes individués. Après avoir réalisé un examen critique de trois tentatives de réponse à cette question l'approche positiviste traditionnelle, celle fondée sur la notion de « modèle » et celle, weberienne, qui fait appel aux « types idéaux " -, nous envisageons cette articulation comme rapport particulier entre concepts théoriques - portant sur des déterminations ou objets abstraits-formels -, et concepts empiriques - portant sur celles de la singularité des objets concrets - Cette solution méthodologique est présentée via la construction d'un concept exemplaire, celui de "formation sociale ", et l'analyse de son fonctionnement dans l'étude d'un cas concret, à savoir les sociétés latino-américaines. 
PHILOSOPHIQues, Vol. XI, Numéro 2, Octobre 1984

\title{
L'ARTICULATION DU GÉNÉRAL ET DU PARTICULIER : UNE APPROCHE MÉTHODOLOGIQUE DANS LE CHAMP DES SCIENCES SOCIALES
}

\author{
Par Roberto Miguelez
}

RÉSUMÉ. La question de l'articulation du général et du particulier n'a pas qu'une dimension épistémologique, elle commande aussi la résolution du problème $\mathrm{du}$ rapport entre théorie et pratique éclairée, et ceci dans la mesure où la pratique s'exerce toujours sur des processus ou des phénomènes individués. Après avoir réalisé un examen critique de trois tentatives de réponse à cette question - l'approche positiviste traditionnelle, celle fondée sur la notion de "modèle " et celle, weberienne, qui fait appel aux « types idéaux »-, nous envisageons cette articulation comme rapport particulier entre concepts théoriques - portant sur des déterminations ou objets abstraits-formels - et concepts empiriques - portant sur celles de la singularité des objets concrets - Cette solution méthodologique est présentée via la construction d'un concept exemplaire, celui de « formation sociale ", et l'analyse de son fonctionnement dans l'étude d'un cas concret, à savoir les sociétés latino-américaines.

ABSTRACT. The articulation of the general and the particular is not a question merely of epistemology. It also comprises a relation between theory and enlightened practice to the extent that processes and individualized phenomena always take place in practice. In this paper we examine critically three approaches to this question of articulation : that of traditional positivism, that based on the notion of "model" and that of Weberian "ideal types". We propose that this articulation is a particular relation between theoretical concepts relating to formal abstract objects or determinations and empirical concepts relating to particular determinations of concrete objects. We illustrate this methodological solution with the concept of "social formation" examining the sense in which it applies to the concrete case of Latin American societies. 


\section{LE PROBLÈME DE L'ARTICULATION DU GÉNÉRAL ET DU PARTICULIER}

1.1. Tout au long de son histoire, et ceci avec plus ou moins de conscience ou d'inconscience, la connaissance sociale et humaine a rencontré le problème de l'articulation du général et du particulier. Problème constitutif car de sa solution dépend la forme ou la structure même de cette connaissance, il se laisse formuler de deux manières, l'une répondant à une articulation faible, voire à la limite à une absence d'articulation, l'autre à une articulation forte. Dans le premier cas, on se demandera comment l'exigence d'universalité ou de généralité que la connaissance sociale et humaine énonce pour ses théories ou constructions théoriques peut être compatible, coexistante, avec l'intention de saisir dans toute leur spécificité, donc dans leur singularité, des processus ou des phénomènes socio-historiques ou humains concrets. Dans l'autre cas, on se demandera comment cette exigence d'universalité ou de généralité peut s'accorder logiquement avec cette intention, c'est-à-dire comment le général peut rencontrer et se rencontrer dans le particulier ou le singulier, et ce d'une manière stricte.

Dans l'un ou l'autre cas il s'agit, par ailleurs, d'un problème décisif non seulement d'un point de vue épistémologique, c'està-dire du point de vue de la construction de la connaissance sociale ou humaine, mais aussi du point de vue de la pratique ou de l'action. En effet, toute action pratique n'a lieu que dans le terrain du particulier, sur des processus ou des phénomènes toujours individués, spécifiques. Ainsi donc, si la connaissance du particulier ou du singulier n'est pas nécessairement ou immédiatement connaissance pratique, la pratique suppose ou devrait supposer, si elle se veut pratique éclairée, la connaissance du singulier. Ce qui voudrait dire que la solution du problème du rapport entre théorie et pratique passe par la solution du problème du rapport entre connaissance du général et appréhension cognitive du particulier ou du singulier.

Dans cet article, nous nous proposons d'abord de passer en revue, plutôt rapidement, quelques réponses majeures données à cette question du rapport entre le général et le particulier. Nous offrirons, ensuite, une solution méthodologique à ce problème via la construction d'un concept qui, dans son exempla- 
rité, peut montrer la possibilité d'une articulation logique entre les deux termes de ce rapport : ce sera le concept de "formation sociale " appartenant au champ des sciences sociales. Nous illustrerons, enfin, ne serait-ce que d'une manière schématique, le fonctionnement concret de cette articulation dans son rapport avec la dimension de la pratique politique, et ce en prenant comme cas l'analyse des sociétés latino-américaines.

1.2. Rien n'illustre mieux le fait qu'il s'agit d'un problème épistémologiquement constitutif, et qui pourtant est loin d'avoir reçu une solution satisfaisante, que les partitions qui, à tous les niveaux, traversent l'espace des disciplines sociales et humaines et trouvent dans cette tension entre le général et le particulier leur raison d'être médiate ou immédiate. Partition intradisciplinaire comme celle qui sépare une ethnographie vouée à l'observation et à la description, au classement, au travail sur le terrain, à la limite ou dans le meilleur des cas, à la " monographie »- du grec monos : seul, unique - , et une ethnologie/ anthropologie visant à une " connaissance globale de l'homme " ${ }^{1}$; partition inter-disciplinaire aussi dont la plus remarquable est sans doute celle qui sépare l'histoire de la sociologie ou, pour reprendre les termes de W. Dilthey, les sciences historiques des "sciences morales systématiques »- dans la mesure où celles-ci, seules, porteraient sur des "systèmes ", c'est-à-dire sur des ensembles structuraux constants permettant le développement de théories générales ${ }^{2}$; partition, enfin, que nous pourrions appeler paradigmatique qui distingue une visée cognitive "idiographique " et une visée cognitive "nomothétique » - dans la terminologie établie par Cournot, reprise par tout l'historicisme néo-kantien et que l'on retrouve encore chez

1. Cf. par exemple, Lévi-Strauss, Claude, Antbropologie structurale, Paris, Plon, 1958, p. $387-$ 88. Il est à signaler cependant que Lévi-Strauss conçoit cette division comme tripartite, l'ethnologie représentant " un premier pas vers la synthèse ", autrement dit vers " des conclusions suffisamment étendues ", et l'anthropologie " tendant à des conclusions, positives ou négatives, mais valables pour toutes les sociétés humaines ", c'est-à-dire de généralité maximale. (Cf. ibid).

2. Cf. Dilthey, W., Introduction à l'étude des sciences bumaines, trad. L. Sauzin, Presses Universitaires de France, Paris, 1942, p. 146, p. 68/70. Dilthey était bien conscient de la dimension du problème que soulevait cette partition. Dans la discussion des thèses de Windelband il désigne, en effet, le problème de la " combinaison du général et de l'individuel " comme le problème logique fondamental des sciences morales systématiques. Cf. Dilthey, W., Le monde de l'esprit, Trad. M. Remy, Paris, Aubier, Éd. Montaigne, 1947 , p. 263. 
K. Popper (et le paradoxe n'est qu'apparent car cette distinction est fondée chez Popper d'une tout autre manière, c'est-à-dire est débarrassée de tout ontologisme) $)^{3}$.

D'où viendrait ce problème constitutif et, plus particulièrement, pourquoi sa solution a pris si souvent et même si systématiquement la forme d'une partition du savoir, voire même et préalablement des visées cognitives censées le sous-tendre ? «Il n'y a de science que du général » affirme déjà Aristote, autrement dit une connaissance théorique de l'individuel est impossible. Certes, cela ne veut pas dire que l'individuel ou le particulier ne puissent pas être connus - c'est, pour Aristote, justement l'objet de la connaissance historique - mais non seulement cette connaissance serait non-théorique et purement descriptive, elle constituerait aussi, et par là même, une connaissance d'un genre inférieur ${ }^{4}$. De sorte que, comme le signale G.G. Granger, nous nous trouvons alors enfermés dans un dilemme : " (. . .) ou bien il y a connaissance de l'individuel, mais elle n'est pas scientifique, ou bien il y a science du fait humain, mais qui n'atteint pas l'individu $»^{5}$. Dilemme d'autant plus redoutable que, dès lors, et dans la mesure où la pratique rencontre son lieu, son objet, dans l'individuel, dans le spécifique, ou bien elle se condamne à être une pratique sans fondement, ou bien à le chercher ailleurs que dans la science.

1.3. Rappelons quelques tentatives de solution de ce problème afin, surtout, de mieux cerner les enjeux qu'il comporte. Nous venons de noter que la pratique rencontre son objet dans l'individuel. S'il en est ainsi, la solution du problème du rapport entre connaissance générale ou théorique et connaissance de l'individuel, passe, pourrait-on dire, par celle du problème du rapport entre théorie et pratique. L'énoncé de ce problème peut recevoir une formulation plus précise si, d'une part, la théorie est définie au moins comme un système d'hypothèses de forme légale de différent niveau de généralité (énoncés nomothétiques),

3. Cf. Popper, Karl, Misère de l'historzcisme, trad. Rousseau, Paris, Plon, 1956, spéc. chap. IV.

4. Elle serait même inférieure à la poésie : "Aussi, écrit Aristote, la poésie est-elle plus philosophique et d'un caractère plus élevé que l'histoire ; car la poésie raconte plutôt le général, l'histoire le particulier ». (Aristote, Poétique, Paris, Les Belles-Lettres, 1932, 1451 b).

5. Cf. Gkanger, Gilles-Gaston, Pensée formelle et sciences de l'bomme, Aubier, Éd. Montaigne, Paris, 1960, p. 185. 
d'autre part la pratique est caractérisée comme une action destinée à produire un certain effet. Dans ce cas, le problème du rapport entre théorie et pratique devient, rigoureusement, celui du rapport entre énoncé nomothétique et usage pratique de l'hypothèse, plus exactement celui du passage d'un énoncé nomothétique à l'énoncé d'une règle d'action.

Ce passage peut alors être conçu comme étant permis par une inférence "pragmatique " fournissant un fondement réel à la règle. En effet, comme le montre $\mathrm{M}$. Bunge, on peut dire en méta-langage $\#$ la formule étant valable pour des lois et des règles élémentaires - que,

$$
\begin{gathered}
« \mathrm{~A} \rightarrow \mathrm{B} \text { fund («B per } \mathrm{A} » \text { vel } \sim \mathrm{B} \text { per } \sim \mathrm{A} ») \text {. } \mathrm{B} \text { per } \mathrm{A} » \\
\text { aeq } « \mathrm{~B} \text { per } \sim \mathrm{A} »)
\end{gathered}
$$

où « $\mathrm{A} \rightarrow \mathrm{B}$ » est un énoncé nomothétique, fund signifie "fonde " ou " est le fondement de », "per » signifie " au moyen de », «vel» représente « ou» et « aeq» signifie « equieficient ${ }^{6}$.

Quel problème soulève cette solution par rapport à la question qui nous préoccupe ici ? Le passage d'un énoncé nomothétique à la règle d'action n'est pas exactement passage du général au singulier mais plutôt reprise, dans le singulier, d'un général. En d'autres termes, la loi rejoint le singulier seulement dans la mesure où le singulier se présente comme un cas de la loi, donc dans la mesure où il a perdu sa spécificité ou singularité. Dans une telle perspective, le singulier ne peut être conçu que comme le point d'intersection d'un nombre infini de lois.

6. Cf. Bunge, Mario, Scientific Research II : The Search for Truth, Springer Verlag, New York, 1967, par. 11.2. Pour mieux visualiser la formule, soit l'exemple suivant :

Énoncé nomologrque : "La rubéole des femmes enceintes ( $\mathrm{A}$ ") entraîne des malformations du fœetus ( $\mathrm{B} »)$.

Règles d'action :

$$
« \mathrm{~A} \rightarrow \mathrm{B} *
$$

R. 1. "Si nous ne voulons pas que le fœetus présente des malformations ( $\sim B$ "), il faut que la femme enceinte ne soit pas atteinte de rubéole $(« \sim A »)$

$$
\text { " } \sim \mathrm{B} \text { per } \sim \mathrm{A} \text { " }
$$

R.2. "Si nous voulons que le fotus présente des malformations («B "), il faut que la femme enceinte soit atteinte de rubéole ( $A$ »).

$$
\text { “B per A " }
$$

Nous examinons exhaustivement le problème du fondement d'une action fondée sur un énoncé nomologique in Miguelez, Roberto, Science, valeurs et rationalité, Presses de l'Université d'Ottawa, Ottawa, 1984, spéc. chap. 1.5 et chap. 2.2. 
Il peut ainsi être décrit mais non pas entièrement expliqué et partiellement décrit, par ailleurs - et ce n'est pas lui non plus qui peut être l'objet d'une action mais seulement certains de ses aspects ${ }^{7}$.

À cette approche, qui correspond au schéma positiviste traditionnel, on peut opposer une perspective qui substitue la notion de "modèle » à celle de « système (de lois) », et qui fait de la pratique non pas un sous-produit éventuel de la connaissance du général mais un facteur décisif de la conceptualisation de l'individuel. G.G. Granger voit même dans une physique capable de prévoir et de modifier l'évolution de complexes déterminés et dans une chimie qui crée des espèces nouvelles en dominant un ensemble de conditions, des percées significatives dans la connaissance et la mâttrise de l'individuel. Or, dans cette évolution, l'aspect méthodologique essentiel serait, pour Granger, justement « le passage de la notion de système - c'est-àdire de schéma visant une description universelle et homogène — à la notion de modèle - c'est-à-dire de schéma d'un complexe relativement autonome, où sont distingués des facteurs stratégiques ${ }^{8}$. Ces modèles, qui, parce qu'ils incluent à titre essentiel la notion de stratégie supposent la pratique comme dimension constitutive, se rencontreraient cependant de préférence, sinon décisivement, dans les sciences humaines. D'où le "pôle clinique " qui caractériserait, selon Granger, ces sciences.

Or, l'individuel comme "irréductible " - non plus déjà, certes, à un "système " mais à un " modèle " - ne disparaît pas pour autant dans une telle perspective. Seulement, au lieu de constituer une barrière par principe infranchissable, il y devient incitation, davantage le lieu et l'objet de la pratique :

L'individuel, en ce sens, marque la limite de la science, mais en apparence seulement, car la science envisagée comme moment de la pratique totale se coordonne tout naturellement et nécessairement à un art $(. . .)^{9}$.

N'empêche que cette dialectique entre savoir et pratique, entre connaissance et art, si elle permet de découvrir des grilles de

7. Cf. par exemple, Hayek, Friedrich von, Scientisme et sciences sociales, Plon, Paris, 1953, spéc. p. $74 / 84$.

8. Cf. Granger, Gilles-Gaston, ibid, p. 199/200.

9. Cf. Granger, Gilles-Gaston, ibid, p. 203. 
décodage de plus en plus fines doit nécessairement postuler l'existence d'une limite radicale de la science et assumer le principe à proprement parler ontologique d'un débordement inexorable de la conduite humaine par rapport à la pratique scientifiquement orientée ${ }^{10}$. Lieu de liberté mais, dès lors, saisie comme incertitude, ce résidu par principe non maîtrisable scientifiquement se révèle alors aussi limite à la prétention d'une pratique complètement fondée, sinon intégralement réfléchie.

On connaît l'importance de la tentative weberienne de résoudre le problème de la saisie cognitive de la singularité et de la spécificité grâce à la construction conceptuelle qu'est le type idéal. Dans ce cas, un ensemble de définitions - qui, pourtant, comme le signale $\mathrm{R}$. Aron ne sont pas conformes au modèle de la logique aristotélicienne ${ }^{11}$ - se substitue aussi au système de lois mais, à la différence du « modèle » chez Granger, son inadéquation inéluctable par rapport à la réalité n'y apparaît pas comme le résultat d'un principe ontologique, limite ou résidu à réduire dans un processus sans terme, mais comme artefact méthodologique : "Il n'a d'autre signification que d'un concept limite (Grenzbegriff) purement idéal, auquel on mesure (messen) la réalité pour clarifier le contenu empirique de certains de ses éléments importants, et avec lesquels on la compare ${ }^{12}$.

Les conséquences méthodologiques d'un tel changement de statut ne sont pas négligeables - la déviation par rapport au type idéal devient, en effet, dès lors, l'enjeu du savoir empirique - Mais ce qui nous intéresse de remarquer est le fait que, pour Weber, le type idéal d'un individu historique ne peut être qu'une reconstruction (idéale) partielle. Et ceci, non pas simplement parce que toute la réalité ne pourrait pas entrer dans l'image mentale du sociologue qui construit le type idéal mais, plus fondamentalement, parce que, pour Weber - et en ceci il ne

10. Cf. Granger, Gilles-Gaston; ibid, p. 218. Nous ne nous attarderons pas, par ailleurs, au problème que, dans cette perspective, soulève l'histoire qui, d'un côté, réapparaît comme connaissance non-scientifique, comme art, d'un autre côté, avec le statut paradoxal d'une " clinique sans pratique".

11. Parce que les types idéaux ne retiennent pas les caractères que présentent tous les individus inclus dans l'extension du concept ni, moins encore, les caractères moyens des individus considérés, mais le typique, l'essentiel. (CF. Aron, Raymond, Les étages de la pensée sociologique, Gallimard, Paris, 1967, p. 520).

12. Cf. Weber, Max, Essais sur la théorie de la science, Paris, Plon, 1965, p. 185. 
faisait qu'emprunter à $\mathrm{H}$. Rickert la perspective néo-kantienne de l'historicisme allemand -, toute reconstruction singularisante l'est pas rapport à des valeurs. Il s'ensuit que, ne seraitce qu'en principe, il y aura autant de perspectives historiques ou sociologiques qu'il y a de valeurs servant à la sélection des aspects ou des caractéristiques en vue de la reconstruction singularisante.

C'est aussi, et dans une large mesure, comme résultat d'une réflexion sur la nature exacte de l'objet du Capital de K. Marx que Weber croit trouver dans le type idéal l'outil méthodologique susceptible d'appréhender les individualités auxquelles s'intéressent ou devraient s'intéresser les sciences sociales et humaines. Et, en effet, c'est à plusieurs reprises dans le Capital que Marx, après avoir caractérisé son étude comme celle du type général (allgemeinen Typus) des rapports économiques réels, définit ce type général comme « moyenne idéale » (idealer Durchschnitt) du mode de production capitaliste: C'est après avoir constaté la présence de ce vocabulaire dans le texte de Marx que L. Althusser propose, à l'encontre de Weber, une interprétation originale de l'objet du Capital en redéfinissant cette idéalité comme « idéelité », c'est-à-dire simple conceptualité de son objet, et la "moyenne " comme contenu du concept de son objet - et non comme résultat d'une abstraction empirique ${ }^{13}$, d'une reconstruction (idéale) partielle, perspectiviste, de la réalité. Ce qui nous intéresse dans cette interprétation c'est le nouveau déplacement de la problématique de la saisie de la spécificité qu'elle rend possible axée dorénavant sur les mécanismes mêmes de la conceptualisation, sur la manière dont la construction de l'objet de la connaissance pourrait permettre, suivant les termes de Marx, l'« appropriation cognitive » du réel - et d'un réel saisi dans sa singularité ou spécificité.

Peu d'indications nous sont fournies par Althusser concernant cette construction ${ }^{14}$ mais elles sont suffisantes ne serait-ce

13. Cf. Althusser, Louis, «L'objet du capital. Appendice : sur la «moyenne idéale» et les formes de transition ", in Althusser, L., Balibar, E., Establet, R., Lire le Capital, Tome II, F. Maspero, Paris, 1965, spéc. p. 179/81.

14. Elles se trouvent rassemblées, au niveau méthodologique qui nous intéresse, dans le seul article "Sur le travail théorique ", La Pensée, No. 132, mars-avril 1967 - bien que certaines de ces indications sont reprises dans l'Avertissement aux lecteurs du Livre I du Capital "(Paris, Garnier, 1969). 
que pour esquisser une nouvelle solution du problème. Althusser part encore du principe suivant lequel «toute connaissance, donc tout discours théorique a pour fin ultime la connaissance de ces objets réels, concrets, singuliers (la France de 1848, la Russie de 1905 ou de 1917, précise Althusser); soit leur individualité (la structure d'une formation sociale), soit les modes de cette individualité (les conjonctures successives dans lesquelles existe cette formation sociale) ${ }^{15}$. La connaissance de ces objets est pensée alors comme le résultat d'un procès conçu comme "synthèse d'une multiplicité de déterminations ", plus exactement, et d'un point de vue méthodologique, comme «combinaison-conjonction » de deux types de concepts : les concepts théoriques (au sens fort) portant sur des déterminations ou objets abstraits-formels, et les concepts empiriques portant sur les déterminations de la singularité des objets concrets. Le rapport entre ces deux types de concepts ne doit pas être vu, propose encore Althusser, ni comme réduction, ni comme déduction, ni comme subsomption des uns aux autres mais comme "réalisation": les concepts empiriques "réalisent " les concepts théoriques dans la connaissance concrète des objets concrets - dans un sens proche de l'expression de Marx parlant de la « réalisation de la plus-value ". Seulement, la dialectique de cette "réalisation " exigerait de longs éclaircissements qui, remarque Althusser, ne peuvent être produits que " sur la base d'une théorie de la pratique des sciences $"{ }^{16}$.

1.4. Or, il est dorénavant possible, sur la base d'une pratique déjà suffisamment riche de l'analyse sociologique d'éclaircir, ne serait-ce que dans une certaine mesure, la dialectique de cette " réalisation » et, par ce chemin, de mesurer la fécondité d'une réponse au problème de la combinaison du général et du singulier reformulée en termes de l'articulation entre déterminations abstraites-formelles et déterminations empiriques.

15. Cf. Althusser, Louis, "Sur le travail théorique », ibid, p. 5. (Souligné par Althusser).

16. Cf. Althusser, Louis, ibid, p. 7. (C'est nous qui soulignons, R.M.). Nous sommes redevables à un des lecteurs de cet article d'avoir signalé la pertinence de l'analyse de Leszek Nowak (The Structure of Idealization. Towards A Systematic Interpretation of The Marxian Idea of Sczence, D. Reidel Publ., Co., Dordrecht, Holland, 1980) par rapport à notre problématique. L'espace nous manque ici pour discuter cette analyse tout à fait remarquable avec laquelle coïncide, dans ses grandes lignes, la nôtre. Disons seulement que notre objectif n'est pas ici, comme chez Nowak, une reconstruction systématique du "modèle idéationnel " ("idealizational model") de la science mais l'examen de la solution originale qui s'en dégage par rapport au problème de l'articulation du général et du particulier. 
La fécondité de cette réponse se mesure à plusieurs séries de raisons. Premièrement, à la différence de la solution positiviste, elle semble bien viser la saisie stricte de la spécificité ou de l'individualité dans la mesure où celle-ci n'est pas appréhendée simplement comme un cas de la loi - la subsomption sous la loi ou le système de lois exigeant la réduction de l'objet réel concret singulier à ses caractéristiques nomologiquement mầtrisables. Deuxièmement, et à la différence d'une perspective en termes de "modèles " ou de "types ", idéaux ou autres, elle ne suppose pas l'existence d'une barrière infranchissable entre l'objet théorique et l'objet réel concret singulier - que ce soit, comme chez Granger, la barrière qui découlerait du débordement de la conduite humaine par rapport à la pratique scientifique, que ce soit, comme chez Weber, celle qui, par principe, sépare la réalité de l'idéalité - Troisièmement, et c'est, peutêtre, la conséquence la plus importante, elle vise à fournir à la pratique autant une explication suffisante qu'une orientation intégrale réfléchie. En acceptant que tout ne peut pas être l'objet d'une action pratique (postulat positiviste anti-totaliste), elle considère cependant qu'une action pratique peut avoir des conséquences sur le tout, et ceci non pas parce qu'elle devrait porter sur des « variables stratégiques " déterminées par les seuls objectifs de l'action (Granger), voire par des choix de valeurs (Weber) mais parce qu'elle pourrait porter, grâce à la connaissance rendue possible par la construction d'objets abstraits-formels, sur ce qui détermine structurellement les objets réels concrets singuliers ${ }^{17}$. Il s'agit d'une solution méthodologique du problème en ce sens que, d'une part, elle implique la construction de concepts sociologiques susceptibles de permettre l'articulation du général et du particulier, d'autre part l'application réglée de ces concepts fournit ou doit fournir autant une connaissance de la spécificité

17. Il s'agirait, pour $\mathrm{L}$. Nowak, de la conséquence décisive d'assumer un postulat ontologique essentialiste, la structure interne d'un phénomème étant représentée, dans la théorie, par les hypothèses portant sur les facteurs ou " grandeurs " essentiels, principaux, ou ayant le plus d'influence (Voir Nowak, L., ibid, p. 95/104). Remarquions, au passage, que L. Althusser avait déjà discuté une interprétation essentialiste de la théorie au nom d'un anti-empirisme et d'un anti-positivisme. (Voir Althusser, L., " Du Capital à la philosophie de Marx ", spéc. par. 10, in Althusser, L., Rancière, J., Macherey, P., Lire le Capital, tome I, F. Maspéro, Paris, 1966). Malgré son importance épistémologique, nous n'examinerons pas cependant cet aspect de la question. 
d'une situation concrète que les fondements d'une pratique (politique). Davantage, cette application réglée doit ou devrait permettre de comprendre pourquoi certaines pratiques (politiques) ont eu, ou ont pu avoir lieu.

1.5. Si nous considérons que les formations sociales sont non seulement le terrain réel de processus socio-historiques spécifiques mais elles-mêmes des objets réels singuliers, l'analyse des formations sociales a une fonction en quelque sorte stratégique dans le traitement de cette problématique. Méthodologiquement, c'est le concept lui-même de "formation sociale " qui devient alors décisif.

Dans l'approche que nous examinons, ce concept est considéré comme "empirique " et construit à l'aide du concept " théorique " de " mode de production ». La notion de " mode de production » est, dans cette perspective, celle d'un objet abstrait-formel en ce sens que rien, dans la réalité, ne correspond à un mode de production. Non pas que le mode de production soit un "type idéal » (Weber), ou un " modèle » mais parce que la réalité ne montre que des combinaisons de modes de production et, du fait de leur combinaison, les modes de production, dans la réalité, subissent des transformations plus ou moins considérables - par rapport à leur construction abstraite-formelle

Les formations sociales acquièrent leur première et principale détermination en tant, justement, que combinaisons de modes de production. C'est en ce sens que les formations sociales " réalisent " les modes de production. Les modes de production sont des objets abstraits-formels hautement complexes de sorte que la structure d'une formation sociale particulière s'avère alors plus complexe encore. Nous ne pouvons pas ici examiner ni la structure de l'objet abstrait-formel "mode de production ", ni celle de modes de production spécifiques ( « capitaliste ", " féodal », de « petits-producteurs », etc. ${ }^{18}$. Par contre, nous pouvons examiner, bien que d'une manière nécessairement sché-

18. Pour une esquisse de la structure de l'objet abstrait-formel « mode de production ", voir Miguelez, Roberto, "Détermination et dominance ", La Revue canadienne de sociologie et d'anthropologie, 19 (4) Novembre 1982, p. 549/75. En ce qui concerne la structure de modes de production spécifiques, la littérature est déjà plus que considérable. 
matique, la méthodologie de cette conceptualisation dans une analyse concrète. Il s'agira donc plutôt d'une illustration de la méthode destinée à montrer comment, concrètement, elle permet d'opérer une articulation forte de trois dimensions essentielles : la connaissance du général, l'appréhension de la spécificité, et le fondement ou la compréhension de pratiques (politiques, à l'occasion). '̀ cette fin, et comme nous l'avons déjà annoncé, nous choisirons comme cas l'analyse des sociétés latinoaméricaines ${ }^{19}$.

\section{SPÉCIFICITÉ ET STRATÉGIES POLITIQUES :}

LE CAS LATINO-AMÉRICAIN

2.1. Le choix de ce cas n'est ni arbitraire ni fondé sur des raisons extra-méthodologiques. En effet, dans l'analyse des processus socio-historiques qui ont eu lieu ou qui ont lieu dans les sociétés latino-américaines nous rencontrons, sous une forme exemplaire, les partitions intra-disciplinaires, inter-disciplinaires et même paradigmatiques qui traversent les disciplines sociales et humaines - et ceci, par ailleurs, dans un contexte global de dépendance, y compris de dépendance culturelle qui a permis de poser en toute clarté la question paradoxale seulement en apparence de la possibilité d'une "sociologie nationale ». (Nous reviendrons sur cette question à la fin de notre étude).

Deux " attitudes » ont présidé à l'analyse des sociétés latinoaméricaines ou ont été largement dominantes : l' « attitude " ethnographique ou ethnologique, et l'« attitude " sociologique. La première a fait des sociétés constituées avant la conquête par les peuples Européens, et de ce qu'il en restait après, son objet d'étude. Avec cette importante précision : ce reste est thématisé par l'ethnographie ou l'ethnologie tant et aussi longtemps qu'il demeure rural. La technique utilisée massivement est celle de la monographie ethnographique classique, c'est-à-dire la présentation ordonnée, systématique, d'un ensemble de données qui prétendent couvrir toutes les dimensions socialement pertinentes. La spécificité de ces sociétés est ainsi censée être pré-

19. Nous insistons sur le fait qu'il ne s'agit nullement d'une contribution nouvelle à la connaissance des formations sociales latino-américaines qui est proposée ici mais d'une analyse de la logique de leur connaissance, et ceci dans le cadre d'une perspective méthodologique particulière et à la lumière d'un certain enjeu philosophique. 
servée sinon garantie par une description qui se veut neutre, et ceci dans la mesure même où elle sépare la collecte d'informations de leur interprétation, et fonde sur cette séparation son objectivité.

À cette fin, la recherche ethnographique s'est doté d'un appareil conceptuel conçu comme système général de catégories non-théoriques à fonction purement classificatoire ${ }^{20}$. Pour ces sociétés, l'ethnologie ou l'ethnographie remplissent le vide créé par l'absence d'histoire, et ceci non pas seulement parce que certaines de ces sociétés sont censées être ou avoir été " ahistoriques » (sociétés de la Forêt, pour l'essentiel) et que, pour d'autres, toute reconstitution historique est à jamais impossible (Hautes Cultures des Andes) mais aussi parce que c'est la seule attitude qui, dans ces cas, est censée pouvoir garantir les droits d'une visée cognitive idiographique, autrement dit saisir leur spécificité.

On sait bien aujourd'hui jusqu'à quel point ces catégories effectivement non-théoriques ${ }^{21}$ sont pourtant bien peu neutres car elles répondent à quelque chose de plus profond qu'à une théorie : à une représentation du monde. On commence à peine cependant à s'apercevoir jusqu'à quel point les descriptions qu'elles commandent peuvent être empreintes d'ethnocentris$\mathrm{me}^{22}$. Car, bien entendu, cette représentation du monde était celle de l'ethnologue ou de l'ethnographe des sociétés conquérantes.

L'autre « attitude», sociologique, fait, par contre, des sociétés constituées après la conquête, et plus précisément des sociétés contemporaines, son objet d'étude. Avec cette importante précision cependant, que c'est le monde urbain qui est plutôt thématisé. Ce qui est alors remarquable par comparaison

20. Une codification de ce système universel a été entreprise par Murdock, Georges P. et al. in Outline of Cultural Materials, New Haven, H.R.A.F. Press, 1961.

21. D'un point de vue strictement épistémologique, toute théorie comporte implicitement ou explicitement un système de catégories à fonction classificatoire mais il serait difficile de prouver que tout système de catégories renvoie à une théorie au sens strict. Certains en concluent, à tort, qu'un système classificatoire peut être neutre, c'est-à-dire indépendant de toute orientation.

22. Pour le traitement ethnocentrique d'un problème apparemment aussi facilement « objectivable » que celui, démographique, de la population des sociétés de la Forêt, voir Clastres, Pierre, La société contre l'État, Les Édirions de Minuit, Paris, 1974, chap. 4. 
avec l'« attitude » ethnographique ou ethnologique, c'est la prétention explicative de cette « attitude »- et non pas simplement descriptive -, et l'usage explicite de théories ou de modèles théoriques ainsi que d'une gamme de techniques d'observation. C'est sur ces modèles que portera notre attention, sur le concept de "formation sociale " qu'ils impliquent ou présupposent, et sur la façon dont ils fonctionnent ou peuvent fonctionner dans l'appréhension de la spécificité de ces sociétés contemporaines de l'Amérique Latine.

2.2. Le premier modèle théorique à être utilisé dans l'analyse des sociétés latino-américaines est celui qu'il est convenu d'appeler le modèle "dualiste ». Il s'agit, par ailleurs, d'un modèle largement dominant non seulement dans l'analyse de ces sociétés mais dans celle de tous les pays dépendants ${ }^{23}$.

L'idée suivant laquelle deux sociétés coexistent en Amérique Latine, l'une arriérée, l'autre moderne, dont les seuls rapports seraient d'opposition et de conflit est repérable déjà au $\mathrm{XIX}^{\mathrm{e}}$ siècle. Certes, il ne s'agit pas encore d'une idée intégrée dans une théorie au sens strict mais, plutôt, d'un élément immédiatement idéologique, c'est-à-dire appartenant au discours politique. N'empêche qu'elle comportait déjà le potentiel explicatif que le modèle théorique essayera par la suite d'exploiter ${ }^{24}$.

Ce modèle se développe dans deux grandes variantes, l'une dans le cadre d'une perspective marxiste, l'autre dans celui d'une perspective sociologique non-marxiste de type académique. Suivant cette dernière variante, les sociétés latino-américaines se caractériseraient par la présence simultanée non pas simplement de deux secteurs économiques distincts mais de deux véritables sociétés, l'une appelée «traditionnelle ", l'autre « moderne $" 25$.

23. "L'utilisation du modèle dualiste comme système explicatif des sociétés des pays dits en voie de développement est devenue un phénomène de plus en plus courant et persistant dans les sciences sociales. Dans la littérature abondante consacrée aux jeunes nations, ce modèle est fréquemment utilisé par les ethnologues, les sociologues et les économistes pour illustrer la spécificité des structures sociales et économiques de ces pays, par rapport à celles des pays industrialisés". C'est de cette manière que débute l'article de Michel de Coster, "Le modèle de la société dualiste dans les sciences humaines ", Cabiers Internationaux de Sociologie, Vol. XLIX, Juillet-Décembre, 1970, p. 69.

24. Dans sa fonction immédiatement idéologique, cette idée a joué un rôle central dans la justification de la politique libérale d'articulation complète au marché mondial des économies latino-américaines dans la deuxième moitié du $\mathrm{XIX}^{\mathrm{e}}$ siècle.

25. Voici la façon on ne peut plus claire dont un économiste exprime cette idée : "Dualism refers to the coexistence of modern and traditional sectors in a society, and corresponds 
Certes, il s'agirait, dans un cas, d'une société fondée sur une économie pré-industrielle, artisanale, familiale, d'autosubsistance, dans l'autre cas d'une société fondée sur une économie de type industriel, technologiquement avancée, organisée sous la forme d'entreprises modernes produisant pour le marché. Mais il s'agirait aussi, sinon surtout - et ceci, suivant généralement l'approche fonctionnaliste d'origine parsonnienne de deux sociétés différenciées par le type d'action sociale, le type de personnalité, le degré de complexité institutionnelle, le système d'attitudes, etc.

Dans la variante marxiste du modèle il n'est pas question, bien entendu, d'une société traditionnelle et d'une société moderne mais d'une société caractérisée par des rapports de production pré-capitalistes et, plus exactement, de type féodal, et d'une société qui serait déjà pénétrée par des rapports sociaux de type capitaliste. Du point de vue de leur dynamique économique, le processus de croissance serait entièrement le fait de la société ou du secteur capitaliste tandis que la société ou le secteur féodal montrerait une tendance à la régression et à une absorption complète par le secteur capitaliste ${ }^{26}$.

Le pendant non-économique de cette double dynamique, l'une progressive et de croissance, l'autre retardataire et régressive, se trouve développé plutôt dans la variante non-marxiste du modèle : les valeurs et les normes de la société traditionnelle non seulement s'opposeraient mais feraient obstacle à la pensée rationnelle moderne orientée vers le changement, le progrès.

Que ce soit dans sa variante marxiste, que ce soit dans sa variante non-marxiste, le modèle dualiste n'est que la transposition sur un plan «synchronique » de la dichotomie « diachronique " de sociétés que presque toute la sociologie académique a conceptualisé à partir de, et sur la seule base de l'expérience historique des sociétés occidentales du centre du système mondial capitaliste, et que la pensée marxiste tradition-

to the now commonplace observation of outsiders that a developing nation is not one but really two". (Anderson, D.A., Developing National Markets : The Thailand Case, M.S.U. Business Topics, Spring, 1969, p. 31).

26. Cf. Kula, W., Théorie économique du système féodal, Paris, Mouton, 1970, p. 8. 
nelle, sur les mêmes bases, ne s'est pas non plus interdit de penser - bien entendu, dans son propre système conceptuel En effet, la société traditionnelle ou féodale ne constituerait, en Amérique Latine, que la phase ou le stage préalable, nécessairement voué à la disparition, d'un processus historique menant à des formes modernes ou capitalistes. Ce que montre l'Europe dans l'évolution de son histoire, l'Amérique Latine le montrerait dans le présent de sa géographie sociale, ce qui est là succession dans le temps apparaît ici comme étalement dans l'espace. Il va sans dire que ce sera cette histoire-là qui, dans le modèle, restituera ici non des états séparés mais le passage d'un état à un autre.

Si nous nous demandons quel est le concept de " formation sociale " que sous-tend ce modèle ou auquel il peut renvoyer, nous voyons que les formations sociales y sont conçues comme coexistence (dynamique) de modes de production sans connexion véritable - si ce n'est celle de leur opposition, de leur conflit pensé comme résistance de l'une à l'absorption par l'autre ou comme entrave de l'une au développement de l'autre - C. Comment la spécificité des formations sociales latino-américaines est-elle saisie dans ce cadre ? Ce qui caractériserait ces formations sociales serait, tout d'abord, le fait que la société ou le secteur moderne ou capitaliste ne constituerait pas le résultat d'un développement autonome de ces formations, et ceci soit parce qu'il a été induit dès l'extérieur et apparaît donc en quelque sorte comme le fruit d'un processus de croissance dont le centre se trouve ailleurs, soit parce qu'il est toujours réglé par un processus extérieur de croissance, soit enfin pour une raison et l'autre ${ }^{27}$.

Dans un développement particulièrement élaboré de la variante non-marxiste du modèle, la transposition sur un plan « synchronique » de la dichotomie « diachronique » de sociétés traditionnelles et modernes résultant d'un processus induit plutôt qu'auto-généré provoquerait une asynchronie, c'est-à-dire un déphasage qui pourrait être aussi bien géographique (région centrale moderne et régions périphériques), institutionnel (coexistence d'institutions correspondant à des "moments " différents), sociologique (coexistence de caractéristiques objectives et

27. Cf., par exemple, De Coster, Michel, ibid, p. 70. 
subjectives appartenant à des phases différentes) et motivationnel (coexistence dans le même individu d'idées, attitudes, etc., correspondant encore à des phases différentes du processus historique $)^{28}$. Dans l'économie générale du modèle, ces phénomènes d'asynchronie ne seraient que des phénomènes en quelque sorte conjoncturels mais ils pourraient cependant avoir une incidence décisive dans l'apparition de phénomènes politiques spécifiques - tout particulièrement les phénomènes de " populisme »et constituer ainsi des «facteurs » explicatifs nécessaires de formes idéologiques et politiques particulières.

De son côté, la variante marxiste du modèle s'attache plutôt aux conséquences économiques et sociales propres au développement d'un secteur capitaliste qui serait toujours réglé de l'extérieur ou, en tout cas, subirait les conséquences de la forme de sa relation au marché mondial. Ce qui aura des répercussions politiques précises, comme nous le verrons immédiatement.

Les déterminations premières et principales de l'objet concret, réel, "formation sociale " qui découlent de son articulation conceptuelle avec l'objet abstrait-formel qu'est le mode de production commandent directement une analyse des phénomènes de différenciation sociale qui, à son tour, constitue le niveau médiateur dans le passage à la compréhension ou à la proposition d'une pratique politique adéquate. Ainsi, la coexistence de deux modes de production ou de deux sociétés sans connexion véritable renvoie à l'idée d'une double différenciation sociale, l'une correspondant à des rapports sociaux de type capitaliste (bourgeoisie/prolétariat), l'autre à des rapports féodaux (oligarchie foncière féodale/paysans dans des rapports de servitude $)^{29}$.

Dans la mesure où cette coexistence est pensée comme processus dynamique qui oppose un secteur féodal retardataire à un

28. Nous nous référons tout particulièrement à la théorie développée par Gino Germani in Política y sociedad en una época de transición, Buenos Aires, 1965. Pour une analyse du phénomène subjectif d'asynchronie, cf. par exemple, le concept de " conscience segmentée " utilisé par Alain Touraine in "Industrialisation et conscience ouvrière à Sao Paulo ", Sociologie du Travail, No 4, octobre-décembre, 1961, spéc. p. 84.

29. Bien entendu, dans la variante non-marxiste il s'agira, comme le dit Parsons, de deux systèmes de stratification nécessitant, chacun, un examen propre. Cf. Parsons, Talcott, "A Revised Analytical Approach to the Theory of Social Stratification", in Bendix, R. et Lipset, S.M., Class, Status and Power. A Reader in Social Stratification, Glencoe, The Free Press, 1957, p. 118/19. 
secteur capitaliste progressif parce que représentant une phase supérieure dans le développement historique ; et dans la mesure où, dans cette variante marxiste, une des conséquences de la forme de la relation au marché mondial serait le fractionnement de la bourgeoisie et la constitution d'une bourgeoisie " nationale " (correspondant à la tendance à un développement autonome du système), la stratégie qui marquera la pratique politique sera naturellement celle d'une alliance des secteurs « populaires " avec la bourgeoisie « nationale » contre l'oligarchie «féodale » et les fractions bourgeoises « liées aux métropoles». Comme on sait, cette stratégie politique d'alliance avec, ou de support à des secteurs « modernes » ou "progressistes » a été largement dominante en Amérique Latine jusqu'aux années soixante approximativement.

2.3. Vers le milieu des années soixante, le modèle dualiste dans sa version marxiste est soumis à une critique radicale. Ce travail critique débouche sur la proposition, par A. Gunder Frank, d'un autre modèle qui s'oppose point par point au modèle dualiste ${ }^{30}$. En effet, pour A. Gunder Frank, les sociétés latino-américaines - sinon toutes les sociétés dépendantes - ${ }^{31}$ présentent une seule structure, celle correspondant aux rapports de production capitalistes, et ceci parce que l'expansion du système capitaliste aurait pénétré effectivement et complètement toutes ces sociétés. Selon A.G. Frank, ce phénomène se serait produit en Amérique Latine déjà au XVI $\mathrm{X}^{\mathrm{e}}$ siècle. Même les secteurs économiques traditionnellement considérés de type féodal comme les plantations ou les hacienda auraient été dès leur début même des entreprises commerciales capitalistes répondant aux demandes du marché national et international et, en conséquence, gérées suivant une rationalité capitaliste.

Deuxième point d'opposition radicale : comme nous l'avons $\mathrm{vu}$, dans le modèle dualiste la dichotomie "diachronique " de sociétés conceptualisée à partir de, et sur la seule base de l'ex-

30. Cf. Gunder Frank, André, Capitalism and Underdevelopment in Latin America, New York, Monthly Review Press, 1967 ; et Latin America : Underdevelopment or Revolution, New York, Monthly Review Press, 1969. Nous disons bien qu'elle débouche sur cette proposition car cette critique radicale est l'objet d'une très vaste polémique préalable.

31. Ou, au moins, les formations sociales africaines car dans L'accumulation dépendante, A. Gunder Frank élargit à celles-ci ce modèle. (Cf. Gunder Frank, André, L'accumulation dépendante, Paris, Anthropos, 1978). 
périence historique des sociétés du centre du système mondial capitaliste est transposée sous une forme « synchronique " dans l'analyse des sociétés dépendantes de l'Amérique Latine. Or, la présupposition d'une telle transposition est une théorie du développement économique comportant une hiérarchie de stades nécessaires et, par conséquent, l'idée suivant laquelle le passage à un stade plus élevé dans la hiérarchie implique nécessairement un développement économique. Ainsi donc cette transposition sur un plan "synchronique " d'une dichotomie "diachronique " n'est possible que sous la condition d'accepter le même modèle de développement dans les sociétés de la "périphérie ", dominées ou dépendantes, que celui des sociétés du " centre ", dominantes. Et c'est à ce point-ci que l'histoire des sociétés dominantes restitue, dans le modèle dualiste, le passage nécessaire d'un état à un autre dans les sociétés dominées. Lorsque le dualisme est rejeté, c'est cette transposition qui, du même coup, est aussi rejetée mais, avec elle, l'idée d'un seul modèle de développement : à la différence des sociétés du " centre " ce serait, suivant la percutante formule, le sous-développement qui se développerait dans les sociétés dépendantes, et ceci à cause même de leur dépendance, de leur statut de sociétés satellites ${ }^{32}$.

Quel est le concept de «formation sociale » que présuppose le modèle de A.G. Frank ? Dans ce modèle, la formation sociale est pensée comme présence absolue d'un mode de production, c'est-à-dire, en fait, comme coïncidence entre la formation et le mode de production. Il est important de remarquer que si ce concept de "formation sociale " se trouve accompagné de la proposition suivant laquelle il y a deux modèles de développement ou deux dynamiques économiques, celle des métropoles et celle des sociétés dépendantes, il suffit d'un seul pas pour concevoir les sociétés

32. La possibilité théorique existe d'une combinaison particulière du modèle dualiste et de celui de $\mathrm{A}$. Gunder Frank. Nous pensons à la possibilité d'accepter la dynamique du modèle de A. Gunder Frank mais dans le cadre national d'une société considérée comme dualiste. Cette possibilité se serait réalisée chez $\mathrm{R}$. Stavenhagen : "Although he (R. Stavenhagen) criticizes the use of this concept, signale A. Cueva, he merely transposes meanings and continues to utilize it. Thus, no longer is the traditional sector responsible for underdevelopment, but rather the modern sector. This is the basis of the 'internal colonialism' idea (. . .)". Cueva, Agustín, "A Summary of 'Problems and Perspectives of Dependency Theory' ", Latin American Perspectives, Vol. III, Number 4, Fall 1976. Et A. Cueva fait surtout référence à Stavenhagen, Rodolfo, "Seven Fallacies about Latin America", in Petras, James et Zeitlin, Maurice (eds.), Latin America : Reform or Revolution?, Greenwich, Connecticut, Fawcett Publ., Inc. 1968. 
dépendantes ou, plus précisément, la dépendance comme un véritable mode de production. Il se peut fort bien que ce soit justement le cas dans le cadre de ce qu'il est convenu d'appeler la " théorie de la dépendance " et c'est, sans doute, le cas par rapport à la théorie du « mode de production colonial " ${ }^{33}$. Dans une telle perspective, le problème de construction d'un ensemble de catégories générales susceptibles de saisir la spécificité ou, dans notre terminologie, la construction d'un objet abstraitformel peut à la limite se dissoudre pour laisser la place à une perspective dans laquelle le cas singulier réel est défini comme étant aussi théoriquement singulier ${ }^{34}$.

Ce concept de «formation sociale " présupposé par le modèle de A. Gunder Frank commande une analyse du processus de différenciation sociale répondant au schéma d'une tendance à la polarisation prolétariat/bourgeoisie avec cette précision politiquement essentielle cependant, à savoir la négation de l'existence d'une bourgeoisie nationale ou, en tout cas, l'affirmation de sa liaison intime avec les métropoles. Dans le cadre d'une telle perspective analytique, la question d'une stratégie de la transformation sociale a pris, surtout entre les années 1965 et 1975, la forme d'une stratégie d'affrontement direct et massif entre les secteurs "populaires" (paysans/classe ouvrière), et l'ensemble des secteurs «bourgeois». Le contraste est éclatant avec la stratégie politique fondée sur le modèle dualiste dans ses deux variantes (constitution de fronts inter-classes en vue de la réalisation de la phase "supérieure " de développement capitaliste, ce qui impliquerait la suppression de secteurs « archaïques» ou «féodaux » par la voie d'une révolution « démocratico-bourgeoise »).

33. Cf. spéc. Cardoso, Ciro Flamarión Santana, "El modo de producción esclavista colonial en América ", in Assadourian, C.S., Cardoso, C.F.S., Ciafardini, J.C. et Laclau, E., Modos de producción en América Latina, Cuadernos de Pasado y Presente/40. Córdoba, Ed. Siglo XXI, Buenos Aires, 1973.

34. C'est le sens de la critique de A. Cueva : "Although it is undeniable that the history of Latin America differs significantly from that of the advanced countries and from the classic development pattern, it is confusing to argue that there are two ideal types of capitalism. In fact, from a Marxist point of view, what you have is not 'patterns' or 'models' but laws, such as the law of development of capitalism, which hold true in Latin America and elsewhere, to be sure within particular historical conditions. Yet, one has to be careful that one does not define each particular case as a theoretically unique one". (Cueva, Agustín, ibid, p. 14). 
2.4. Dans un travail publié en 1971 sous le titre "Feudalism and Capitalism in Latin America"35, Ernesto Laclau soumet, à son tour, le modèle de A. Gunder Frank à une critique dont les répercussions épistémologiques et méthodologiques sont de la plus grande importance dans notre problématique. Selon cette critique, la conception de A. Gunder Frank comporterait une erreur théorique décisive : un concept non acceptable de « capitalisme " parce qu'essentiellement caractérisé comme un système de production pour le marché au lieu d'être pensé comme un mode de production défini par le type de rapport social de production. Laclau choisit donc de réexaminer les déterminations de l'objet abstrait-formel, c'est-à-dire sa construction même, préalablement à celle de la formation sociale. Et pour cause car, comme nous le verrons maintenant, les déterminations de l'objet concret, réel, en sont dépendantes.

Le caractère décisif de l'erreur de A. Gunder Frank en ce qui concerne l'analyse des sociétés latino-américaines consisterait en ce que, sur la base d'une telle caractérisation, la simple présence de capital (commercial) serait considérée comme le signé de la présence d'un capitalisme - ce qui, par ailleurs, ferait croire que les sociétés latino-américaines ont pu être capitalistes dès la période de leur conquête, c'est-à-dire dès le $\mathrm{XVI}^{\mathrm{e}}$ siècle. Non seulement la production pour un marché ne constituerait pas une caractérisation acceptable du mode de production capitaliste, elle pourrait même contribuer à accentuer et consolider un système féodal au lieu de le désintégrer.

Et c'est là, justement, que se trouve le point crucial à partir duquel Laclau se démarque autant du modèle dualiste que du modèle de A. Gunter Frank car, dans cette perspective, affirmer le caractère féodal des rapports de production dans le secteur agraire ne suppose pas nécessairement la thèse dualiste. En effet, comme nous l'avons vu, le dualisme implique non seulement l'existence de deux secteurs ou sociétés sans connexion véritable mais leur affrontement, leur opposition. Or, dans cette nouvelle perspective, la modernité d'un secteur s'avère être une fonction de l'articulation de l'autre, les deux secteurs sont liés par des rapports, et par des rapports complémentaires. Ainsi, si dans le

35. Cf. Laclau, Ernesto, "Feudalism and Capitalism in Latin America", New Left Review, No 67, 1971. L'article a été repris, avec une postface, in Politics and Ideology in Marxist Theory, N.L.B., London, 1977. 
modèle dualiste la formation sociale est conçue comme coexistence de modes de production et dans le modèle de A. Gunder Frank comme coüncidence avec le mode de production, le concept de «formation sociale " présupposé par l'analyse de Laclau semble bien être celui d'une articulation de modes de production. Il s'agirait, par ailleurs, d'une articulation à dominance dans laquelle la dominance du mode de production capitaliste serait assurée, reproduite, par la survivance de modes de production précapitalistes. A son tour, cette survivance ne serait que l'effet de la dominance du mode de production capitaliste. Précision importante : cette articulation ne pourrait pas être considérée comme étant exactement la même dans toutes les formations sociales latino-américaines, ni identique partout au sein de ces formations. Ce qui caractériserait justement chaque formation sociale particulière serait alors la forme particulière de cette articulation.

Dans le cadre d'une telle perspective, l'analyse des processus de différenciation sociale ne peut que prendre la forme d'une analyse des effets différentiels au niveau des classes et des fractions de classe dus à la forme particulière de l'articulation dańs chaque formation sociale. À son tour, une stratégie de transformation répondant à une telle perspective ne peut être élaborée qu'à la suite d'une analyse de ces effets différentiels au niveau des classes et des fractions de classe, autrement dit elle ne peut être qu'une stratégie spécifique pour chaque formation sociale particulière, voire même une combinaison de stratégies locales.

Il est possible de considérer le passage d'un modèle d'analyse à un autre et, corrélativement, d'un concept à un autre de la formation sociale comme des percées successives dans l'appréhension théorique de ce que ces sociétés ont de particulier ou de spécifique. La spécificité ou la particularité s'avère ainsi " propriété " repérable, définissable et définissante, et non pas résidu "inappréhensible » ou point de fuite des lignes de la détermination. En effet, dans le cadre du modèle dualiste et du concept de formation sociale comme coexistence de modes de production, cette spécificité est saisie sous la forme simple d'état temporel du système par rapport à une séquence universelle - car la présence d'une société traditionnelle ou d'un mode de production pré-capitaliste à côté d'une société moderne ou d'un 
mode de production capitaliste n'y est pensée que comme retard et définit ainsi un moment ou une phase de "sous-développement ».Dans le cadre du modèle de A. Gunder Frank et du concept de formation sociale comme coïncidence avec le mode de production, cette spécificité est saisie comme mécanisme spécifique par rapport à celui des sociétés métropolitaines ou du " centre " - et définit ainsi non pas un moment ou une phase mais une condition ou une dynamique particulière de sous-développement. Enfin, dans le cadre d'un modèle comme celui qui se dégage de la critique de Laclau et du concept de formation sociale comme articulation de modes de production, cette spécificité est saisie comme forme, comme configuration singulière dont les effets ne peuvent être autres que ceux résultant de la singularité de la configuration. Est-ce à dire que, dans ce dernier modèle, nous trouvons réunies les conditions formelles suffisantes de l'appréhension de la spécificité d'une formation sociale?

Que ce soit dans le modèle de A. Gunder Frank, que ce soit dans celui qu'esquisse la critique de Laclau, le concept de " mode de production " réfere à des "facteurs " de nature exclusivement économique ${ }^{36}$. En fait, la notion d' « articulation de modes de production " ne sert pas exactement chez Laclau à construire le concept de " formation sociale » mais plutôt celui de "système économique ». Chez Laclau, le mode de production est caractérisé comme un concept abstrait et les systèmes économiques comme des systèmes concrets de relations constitués par l'articulation de différents modes de production. En ce sens, " tout progrès vers le concret, peut affirmer Laclau, implique une transition analytique progressive allant des modes de production vers les systèmes économiques. Évidemment, ajoute-t-il cependant, l'analyse demeure encore abstraite ; le pas final vers le concret exige que le système économique soit situé en relation avec les niveaux politique et idéologique qui caractérisent une formation sociale déterminée ${ }^{37}$. Dans le contexte de notre problématique, ce pas final vers le concret, vers l'appréhension d'un concret qui se donne toujours comme spécificité

36. Dans la postface écrite par E. Laclau en 1977 , il est dit qu'il s'agit d'une notion inadéquate mais aucune précision n'est donnée concernant la nature de cette inadéquation. Cf. Laclau, Ernesto, ibid, p. 42.

37. Cf. Laclau, Ernesto, ibid. 
exigerait plutôt de concevoir la formation sociale non pas comme articulation simple de modes de production au sens restreint du terme, c'est-à-dire au sens de "systèmes économiques " d'un certain type, mais comme articulation complexe de déterminations économiques et extra-économiques. L'appréhension de la spécificité passerait alors par la construction d'un objet abstraitformel suffisamment riche en déterminations. D'une manière générale c'est, pouvons-nous dire, la richesse de la théorie qui rend réellement possible la saisie de la particularité ainsi qu'une pratique adéquate. Loin d'être un art acquis par simple expérience, voire un comportement dicté par l'intuition, une pratique adéquate, politique ou autre, ne peut ainsi être que pratique informée par la théorie, et par une théorie suffisamment riche ; ne peut être que le produit d'une articulation adéquate du général et du particulier.

Dans ce cas, la question d'une sociologie nationale cesse, par ailleurs, de revêtir l'apparence d'un non-sens. Elle se définit comme tentative de saisir la singularité d'une formation sociale concrète, comme repérage systématique de ses déterminations propres, et cette tentative ne s'oppose point à la démarche théorique de construction d'objets abstraits-formels mais, au contraire, apparaît comme son aboutissement nécessaire, comme sa raison d'être même.

Département de sociologie et de philosophie Université d'Ottawa 


\section{OUVRAGES CITÉS}

ALTHUSSER, Louis, «Du Capital à la philosophie de Marx ", in Althusser, L., Rancière, J., Macherey, P., Lire le Capital, tome I, F. Maspéro, Paris, 1966.

"L'objet du Capital ", in Althusser, L., Balibar, E., Establet, R., Lire le Capital, tome II, F. Maspéro, Paris, 1965.

"Sur le travail théorique », La Pensée, No. 132, mars-avril, 1967.

ANDERSon, D.A., Developing National Markets : The Thailand Case, M.S.U., Business Topics, Spring 1969.

ARIStote, Poétique, Paris, Les Belles-Lettres, 1932.

ARON, Raymond, Les étapes de la pensée sociologique, Gallimard, Paris, 1967. BUNGE, Mario, Scientific Research II : The Search for Truth, Springer Verlag, New York, 1967.

CARdoso, Ciro F.S., «El modo de producción esclavista colonial en América ", in Assadourian, C.S., Cardoso, C.F.S., Ciafardini, J.C., Laclau, E., Modos de producción en América Latina, Cuadernos de Pasado y Presente/40, Cordoba, Ed. Siglo XXI, Buenos Aires, 1973.

Clastres, Pierre, La société contre l'État, Les Éditions de Minuit, Paris, 1974. Cueva, Agustin, "A Summary of 'Problems and Perspectives of Dependency Theory" ", Latin American Perspectives, Vol. III, Number 4, Fall, 1976.

DE COSTER, Michel, "Le modèle dualiste dans les sciences humaines », Cabiers Internationaux de Sociologie, Vol. XLIX, Juillet-Décembre, 1970.

DILTHEY, W., Introduction à l'étude des sciences humaines, Trad. L. Sauzin, Presses Universitaires de France, Paris, 1942.

Le monde de l'esprit, Trad. M. Remy, Paris, Aubier Éd. Montaigne, 1947.

FRANK, André Gunder, Capitalism and Underdevelopment in Latin America, New York, Monthly Review Press, 1967.

Latin America : Underdevelopment or Revolution, New York, Monthly Review Press, 1969.

L'accumulation dépendante, Paris, Anthropos, 1978.

Germani, Gino, Política y sociedad en una época de transición, Buenos Áires, 1965.

Granger, Gilles-Gaston, Pensée formelle et sciences de l'homme, Aubier Éd. Montaigne, Paris, 1960.

HAYEK, Friedrich von, Scientisme et sciences sociales, Plon, Paris, 1953.

KULA, W., Théorie économique du système féodal, Paris, Mouton, 1970.

LACLAU, Ernesto, "Fascism and Ideology", in Politics and Ideology in Marxist Theory, N.L.B., London, 1977.

"Feudalism and Capitalism in Latin America", New Left Review, No. 67, 1971. Repris in Politics and Ideology in Marxist Theory, ibid.

Levi-STRauss, Claude, Antbropologie structurale, Plon, Paris, 1958.

MIGUELEZ, Roberto, "Détermination et dominance ", La revue canadienne de sociologie et d'anthropologie, 19 (4), 1982.

Science, valeurs et rationalité, Presses de l'Université d'Ottawa, Ottawa, 1984. 
MURDOCK, Georges et al., Outline of Cultural Materials, New Haven, H.R.A.F. Press, 1961.

NOWAK, Leszek, The Structure of Idealization.Towards A Systematic Interpretation

Of The Marxian Idea of Science, D. Reidel Publ. Co., Dordrecht, Holland, 1980.

PARSONS, Talcott, "A Revised Analytical Approach to the Theory of Social Stratification", in BENDIX, R., LIPSET, S.M., Class, Status and Power. A Reader in Social Stratification, Glencoe, The Free Press, 1957. POPPER, Karl, Misère de l'bistoricisme, Trad. Rousseau, Plon, Paris, 1956. Stavenhagen, Rodolfo, "Seven Fallacies about Latin America", in PETRAS, J., ZEITLIN, M. (eds.), Latin America : Reform or Revolution?, Greenwich, Connecticut, Fawcett Publ. Inc., 1968.

TOURAINE, Alain, "Industrialisation et conscience ouvrière à Sao Paulo ", Sociologie du Travail, No. 4, Octobre-Décembre, 1961.

WEBer, Max, Essais sur la théorie de la science, Plon, Paris, 1965. 